\title{
Expression of BCL6 in paediatric B-cell acute lymphoblastic leukaemia and association with prognosis
}

\author{
Artturi Mäkinen ${ }^{1,2}$, Atte Nikkilä ${ }^{1}$, Juha Mehtonen ${ }^{3}$, Susanna Teppo ${ }^{1}$, \\ Laura Oksa ${ }^{1}$, Jessica Nordlund ${ }^{4}$, Samuli Rounioja ${ }^{5}$, Virva Pohjolainen ${ }^{2}$, \\ SaAra Laukkanen ${ }^{1}$, Merja Heinäniemi ${ }^{3}$, Timo PaAvonen ${ }^{2,6}$, Olli Lohi ${ }^{1,7}$ \\ ${ }^{1}$ Faculty of Medicine and Health Technology, Tampere Center for Child Health Research, \\ Tampere University, Tampere, Finland; ${ }^{2}$ Fimlab Laboratories, Department of Pathology, \\ Tampere University Hospital, Tampere, Finland; ${ }^{3}$ Institute of Biomedicine, School of Medi- \\ cine, University of Eastern Finland, Kuopio, Finland; ${ }^{4}$ Department of Medical Sciences, \\ Molecular Medicine and Science for Life Laboratory, Uppsala University, Uppsala, Sweden; \\ ${ }^{5}$ Fimlab Laboratories, Department of Hematology, Tampere University Hospital, Tampere, \\ Finland; ${ }^{6}$ Department of Pathology, Faculty of Medicine and Health Technology, Tampere \\ University, Tampere, Finland; ${ }^{7}$ Tays Cancer Centre, Tampere University Hospital, Tampere, \\ Finland
}

\begin{abstract}
Summary
B-cell lineage acute lymphoblastic leukaemia (B-ALL) is the most common paediatric malignancy. Transcription factor B-cell lymphoma 6 (BCL6) is essential to germinal centre formation and antibody affinity maturation and plays a major role in mature B-cell malignancies. More recently, it was shown to act as a critical downstream regulator in pre-BCR+ B-ALL. We investigated the expression of the BCL6 protein in a population-based cohort of paediatric BALL cases and detected moderate to strong positivity through immunohistochemistry in $7 \%$ of cases $(8 / 117)$; however, only two of eight BCL6 cases (25\%) coexpressed the ZAP70 protein. In light of these data, the subtype with active pre-BCR signalling constitutes a rare entity in paediatric B-ALL. In three independent larger cohorts with gene expression data, high BCL6 mRNA levels were associated with the TCF3-PBX1, Ph-like, NUTM1, MEF2D and PAX5-alt subgroups and the 'metagene' signature for pre-BCR-associated genes. However, higher-than-median BCL6 mRNA level alone was associated with favourable event free survival in the Nordic paediatric cohort, indicating that using BCL6 as a diagnostic marker requires careful design, and evaluation of protein level is needed alongside the genetic or transcriptomic data.
\end{abstract}

Key words: Acute lymphoblastic leukaemia; B-cell lineage; BCL6; immunohistochemistry; mRNA expression; paediatric.

Received 3 November 2020, revised 30 January, accepted 15 February 2021 Available online 26 May 2021

\section{INTRODUCTION}

Acute lymphoblastic leukaemia (ALL) is the most common malignancy in childhood. Although the long term survival has improved to $80-90 \%$, several smaller subgroups have inferior outcomes. ${ }^{1,2}$ Therapy response at the end-of- induction (EOI) measured by minimal residual disease (MRD) is the most important prognostic factor. ${ }^{1-3}$ However, two major problems remain: first, relapses are still the major cause of mortality, and second, acute and long term side effects are experienced by many survivors due to toxicity in healthy tissues caused by chemotherapy. ${ }^{1}$ Therefore, there is a need for new predictive biomarkers to better identify patients who either could be cured with less intensive treatments or could benefit from the novel targeted agents. 1,2

B-cell lymphoma 6 (BCL6) is a zinc finger containing transcriptional repressor that is essential for germinal centre formation and plays an important role in the somatic hypermutation of immunoglobulin genes. ${ }^{4-7}$ In diffuse large B-cell lymphoma, the BCL6 protein is a central oncoprotein. ${ }^{8}$ BCL6 is highly expressed and is a potential drug target in follicular and Burkitt lymphoma. ${ }^{8}$ In 2015, Geng et al. reported a new subgroup of ALL (pre-BCR+ ALL) with active pre-B-cell receptor (pre-BCR)-signalling marked by high expression of BCL6, and the authors suggested that it constitutes approximately $10-15 \%$ of paediatric and adult ALL cases.' Similar proportions of BCL6 expression have been reported by others. ${ }^{10}$ The subgroup has been associated with the TCF3-PBX1 subtype, and a CD34-negative immunophenotype. $^{9-11}$ Interestingly, pre-BCR+ leukaemia cells were found to be sensitive to tyrosine kinase inhibitors targeting the SYK, SRC, PIK3delta and BTK kinases, 9,12 which highlights the potential clinical utility of the novel subtype. Recently, Hurtz et al. ${ }^{13}$ discovered that BCL6 could serve as a therapeutic target in KMT2A-rearranged leukaemia $(K M T 2 A)$, while Tsuzuki et al. ${ }^{14}$ identified BCL6-positive cases associated with the $M E F 2 D$-translocated ALL $(M E F 2 D)$ and identified the SREBF1 protein in the regulatory network, thus serving as an additional potential drug target in this BCL6-positive entity. Finally, high BCL6 mRNA has been associated with mutations of PAX5 and $I K Z F 1$ and inferior clinical outcomes in high-risk ALL. ${ }^{13,15,16}$ 
The prevalence and clinical significance of BCL6 expression in childhood ALL is unclear, as studies with solely paediatric cases have not been conducted. Here, we studied a population based cohort of paediatric patients with bone marrow (BM) trephine biopsy samples, and utilised three distinct gene expression cohorts (the PanALL study, a Nordic cohort and a collection of studies in the Hemap resource) to investigate the expression of the BCL6 protein and mRNA in B-cell acute lymphoblastic leukaemia (B-ALL). Moreover, we explored the clinicopathological features and patient survival associated with BCL6 expression.

\section{MATERIALS AND METHODS}

\section{Bone marrow biopsies and associated clinical data}

We collected a retrospective patient cohort that included 117 paediatric precursor B-ALL patients who were treated at Tampere University Hospital between 1 January 2000 and 16 October 2017. Only cases under 18 years of age at diagnosis were included. Acute myeloid leukaemia, Burkitt leukaemia, T-cell lymphoblastic leukaemia, and all cases with either inadequate or plastic-embedded biopsy samples were excluded from the cohort. The primary diagnosis was based on the BM aspirate morphology, immunophenotyping and various cytogenetic and molecular genetic investigations. Patients were treated using successive Nordic Society for Pediatric Hematology and Oncology (NOPHO) ALL protocols. Associated clinical data included the following parameters: age, white blood cell count (WBC), blast count, immunophenotype by flow cytometry, cytogenetics and genetics information, involvement of the central nervous system at diagnosis, MRD at EOI, relapse data, secondary malignancies and death during the follow-up period. Subgroup allocation was performed according to the World Health Organization (WHO) 2017 classification criteria. ${ }^{17}$ For cases lacking the genetic subtype information, fluorescence in situ hybridisation analysis was performed on either BM aspiration samples or formalin fixed and paraffin embedded (FFPE) samples. ${ }^{18}$

\section{Immunohistochemical analysis of bone marrow biopsies}

Decalcified (ethylenediaminetetraacetic acid) and FFPE histological BM trephine biopsy samples were collected from the pathology archives from the time of the primary diagnosis. Whole tissue sections $4 \mu \mathrm{m}$ thick were stained with the primary antibodies against BCL6 (clone LN22, PA0204, mouse monoclonal, lot number 48794, dilution 1:50; Leica Biosystems, UK), pSTAT5-Y694 (clone E208, ab32364, lot number GR208043, dilution 1:50; Abcam, UK), ZAP70 (clone YE291, ab32429, rabbit monoclonal, lot number GR59787, dilution 1:100; Abcam) and muHC (clone A0425, rabbit polyclonal, lot number 00061133, dilution 1:5500; Dako, Denmark). Immunohistochemical staining was performed using the Ventana Benchmark Classic instrument (Ventana, USA). The Ultraview Universal DAB Detection kit (Ventana) and haematoxylin counterstain were used for antibody and cell detection, respectively. Appendix, tonsil, invasive ductal breast carcinoma and Burkitt lymphoma/leukaemia were used as control material. The immunohistochemically stained tissue sections were analysed independently by two pathologists using a light microscope. Positive staining in over $50 \%$ of the blast cell nuclei or cytoplasm were graded as strongly positive, $20-50 \%$ as moderately positive, and less than $20 \%$ as negative. Discrepant cases were resolved by a third pathologist.

\section{Gene expression datasets}

Three independent RNA expression datasets were used to analyse BCL6 gene expression as previously described: ${ }^{18}$ (1) the Hemap dataset, which is a microarray dataset of 36 haematological malignancies that includes 6832 samples, including 662 paediatric and 642 adult B-ALL cases collected from different original studies that represent both high risk cohorts and those that include also common good prognosis subtypes; ${ }^{19,20}$ (2) the PanALL study dataset, an RNA-sequencing dataset $(n=1988)$, which includes 1234 paediatric and 754 adult B-ALL cases from different patient cohorts and therapy risk groups; ${ }^{21}$ (3) a Nordic dataset, which consists of RNA-sequencing data from 115 paediatric B-ALL cases that represent cases diagnosed between the years 1996 and 2010 from different therapy risk groups. ${ }^{22}$ The pre-BCR 'metagene' signature was studied in the Hemap and PanALL datasets, and included IGLL1, IGLL3, VPREB1, VPREB3, IGHM, SYK and ZAP70 genes, as described by Geng et al. ${ }^{9}$

Normalisation of the mRNA expression values has been described previously for the Hemap dataset ${ }^{19,20}$ and the PanALL dataset. ${ }^{21}$ Briefly, 'normalised expression values' in the Hemap microarray data ${ }^{19,20}$ were normalised with RMA (robust multi-array analysis) probe summarisation algorithm and corrected for technical bias before log2-transformation. In the PanALL dataset, ${ }^{21}$ 'normalised expression values' were corrected for batch effect.

\section{Ethical considerations}

The study was approved by the local ethical committee (Pirkanmaa Hospital District Ethical Committee, R16054 and R13109) and the National Supervisory Authority for Welfare and Health (Valvira, Dnro:4243/06.01.03.01/ 2016).

\section{Survival and statistical analysis}

Kaplan-Meier survival analysis was used to associate the biological findings with overall survival (OS) and event-free survival (EFS). The events that were factored into the EFS variable were death, relapse, resistant disease (MRD $>25 \%$ at EOI) and secondary malignancy. Cox regression models were used to estimate hazard ratios (HRs) for EFS using the BCL6 mRNA status (median expression as a cut-off), age, WBC, treatment group and cytogenetic subgroup as variables. Proportionality assumptions of the different time dependent variables were tested. The pre-BCR 'metagene' was formed as the arithmetic mean of the pre-BCR associated genes.

Statistical analysis was performed using SPSS Statistics (version 26; IBM, USA) and RStudio (version 3.6.1; RStudio, USA). The Mann-Whitney U and Kruskal-Wallis $\mathrm{H}$ tests were used to analyse continuous non-parametric variables, while the chi-squared test was applied on categorical variables and Spearman's rank order test for correlation. $p$ values less than 0.05 were considered statistically significant, and all statistical tests were two-sided.

\section{RESULTS}

\section{Clinicopathological features associated with BCL6 immunostaining}

BCL6 is a transcriptional repressor that plays a major role in mature B-cell lymphoma. ${ }^{8}$ Recently, BCL6 has been shown to contribute significantly to the pathogenesis of acute precursor B-cell acute leukaemia. ${ }^{9,23}$ We investigated the expression of the BCL6 protein in childhood B-ALL by collecting trephine biopsy samples from 117 paediatric BALL cases. The cohort was population-based, and the main B-ALL subtypes were represented at expected proportions (Table 1). ${ }^{17}$ During the follow-up period [median 8.3 years, interquartile range (IQR) 7.8 years], 15 relapses, nine deaths and two secondary malignancies were registered. Four patients had resistant disease with a blast count of over $25 \%$ at end induction.

Immunohistochemical staining of diagnostic biopsy samples was performed using an antibody against the BCL6 protein. As shown in Fig. 1, the BCL6 protein was expressed with varying intensity in the nuclei of $20-50 \%$ of lymphoblasts. Overall, BCL6-positive cells showed an evenly distributed staining pattern in the leukaemic BM microenvironment so that an approximately equal number of BCL6positive cells were in close proximity of vasculature, bone trabeculae and other stromal cells (Fig. 1). Myeloid background staining was predominantly evident in the granulocytes.

We categorised BCL6 expression based on the staining intensity as either negative or positive (moderate or strong), and the cases were classified independently by two pathologists. With this categorisation, eight of the $117(6.8 \%)$ 
Table 1 Case summary for the patient cohort analysed by immunostaining

\begin{tabular}{ll}
\hline Age, years, median (range) & $4.2(0.9-17.6)$ \\
WBC $\times 10^{9} / \mathrm{L}$, median (range) & $7.1(1-311)$ \\
MRD EOI $(\%)$, median (range) & $0.02(0-44)$ \\
Deceased, $n(\%)$ & $9(7.7)$ \\
Relapsed, $n(\%)$ & $15(12.8)$ \\
CNS disease, $n(\%)$ & $6(5.1)$ \\
SMN, $n(\%)$ & $2(1.8)$ \\
WHO subgroup & \\
ETV6-RUNX1, $n(\%)$ & $33(28.2)$ \\
HeH, $n(\%)$ & $30(25.6)$ \\
$K M T 2 A, n(\%)$ & $5(4.3)$ \\
Ph, $n(\%)$ & $2(1.7)$ \\
TCF3-PBX1, $n(\%)$ & $4(3.4)$ \\
Hypodiploid, $n(\%)$ & $1(0.9)$ \\
Other, $n(\%)$ & $42(35.9)$ \\
Total, $n$ & 117 \\
\hline
\end{tabular}

Age, age at diagnosis; $\mathrm{CNS}$, central nervous system; $\mathrm{HeH}$, high hyperdiploid; MRD EOI, minimal residual disease at the end of induction therapy; $\mathrm{Ph}$, Philadelphia chromosome (BCR-ABL1); SMN, secondary malignant neoplasm; WBC, white blood cell count at diagnosis.

patients were positive for the BCL6 protein (Table 2). Three of four TCF3-PBX1 cases were positive for BCL6 (chisquared $p<0.001)$, in agreement with previous work. ${ }^{10}$ Statistical association with surface markers further confirmed a more differentiated phenotype: four of seven BCL6-positive cases (57\%) were negative for the CD34 stem/progenitor cell marker expression when assessed by flow cytometry at the time of diagnosis, while the majority of BCL6 negative cases were CD34 positive (96/101, chisquared $p=0.001)$. No other surface markers showed a statistically significant association with the BCL6 protein (data not shown).

\section{ZAP70 protein and pSTAT5 are commonly expressed in $\mathbf{B}$-ALL}

Expression of BCL6 has been suggested as a biomarker for the pre-BCR+ subgroup of ALL that has a concomitant high expression of components of the pre-BCR complex and downregulation of the interleukin-7 receptor pathway with downstream STAT5 activity. ${ }^{9}$ We performed immunohistochemical staining of the phosphorylated form of STAT5pY694 (pSTAT5) to assess its exclusivity with the BCL6 protein in B-ALL. The pSTAT5 was seen in 108 (92\%) cases with staining in both the nuclei and cytoplasm of the leukaemia blasts (Fig. 1, Table 2). Of the eight BCL6positive cases, seven were co-expressing pSTAT5 (Table 2). Immunostaining of the heavy chain component of the immunoglobulin receptor (muHC) was hampered by strong background staining of the serum. However, no association was found between the BCL6 protein level and cytoplasmic muHC expression, as assessed by flow cytometry (data not shown).

The ZAP70 protein was expressed mostly in the cytoplasm and present in 110 of 117 (94\%) B-ALL cases. Surprisingly, only two of eight BCL6-positive cases showed simultaneous immunostaining with the ZAP70 protein.
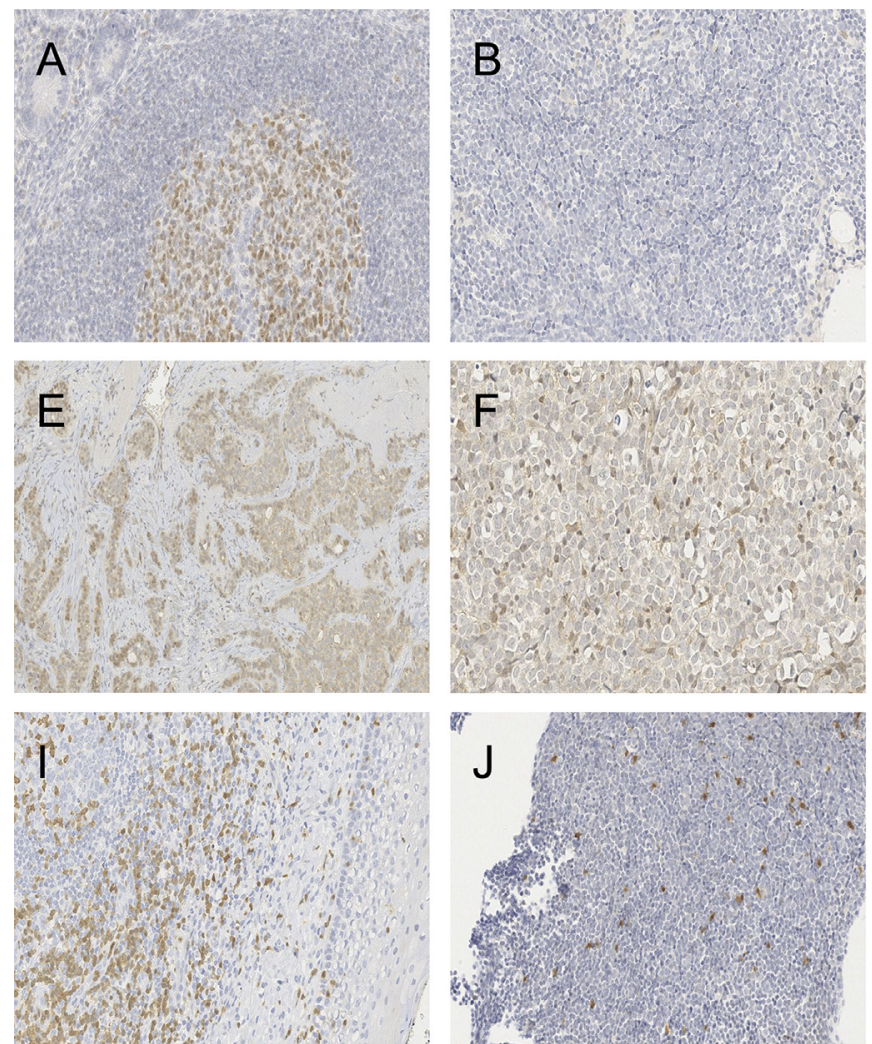
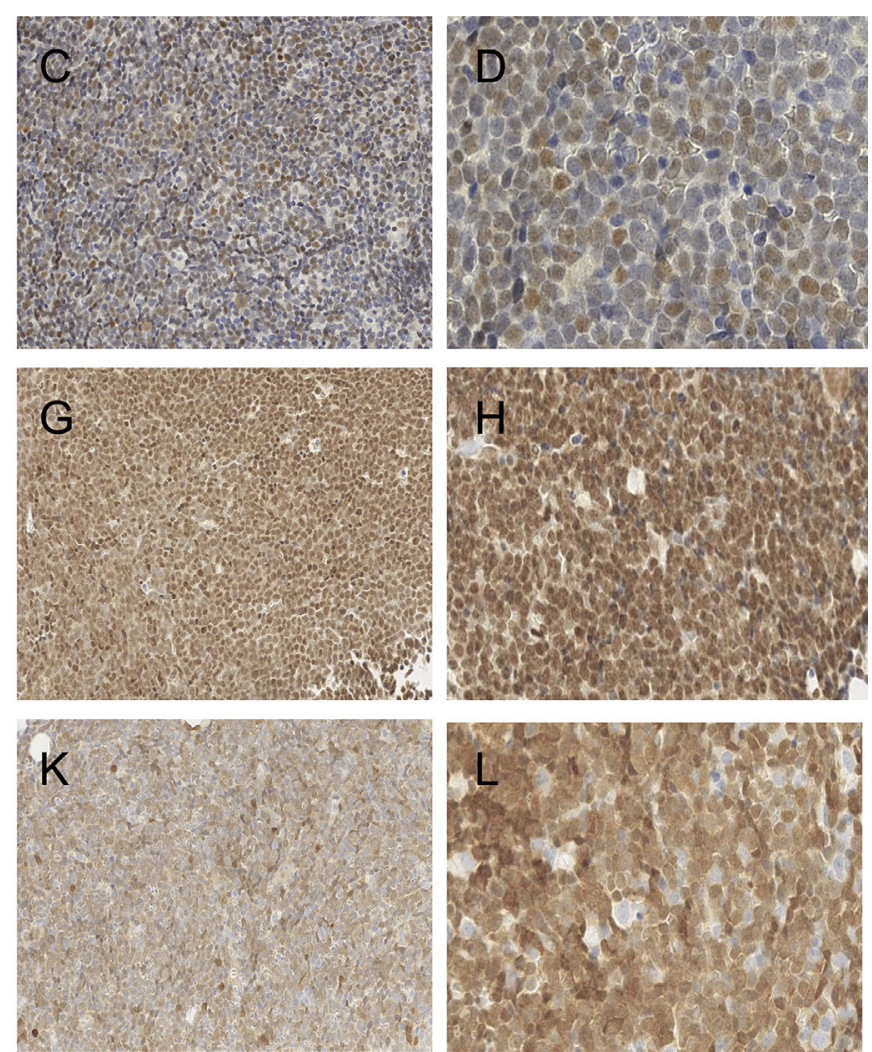

Fig. 1 Immunohistochemical staining of the diagnostic B-ALL trephine biopsy samples. (A-D) BCL6 staining in an appendix (positive control), a negative B-ALL case and two positive B-ALL cases, respectively. (E-H) Staining of the phosphorylated form of STAT5 (Y694) in an invasive ductal breast carcinoma (positive control), a negative B-ALL case and two positive B-ALL cases, respectively. (I-L) The ZAP70 protein immunostaining in the tonsil (positive control), a negative B-ALL case and two positive B-ALL cases, respectively. 
Table 2 Expression of the BCL6, phospho-STAT5 (Y694) and ZAP70 proteins by immunohistochemistry in B-ALL WHO subgroups

\begin{tabular}{|c|c|c|c|c|c|c|c|c|c|}
\hline & ETV6-RUNX1 & $\mathrm{HeH}$ & $K M T 2 A$ & TCF3-PBX1 & $\mathrm{Ph}$ & Hypodiploid & Other & Total & $\%$ \\
\hline \multicolumn{10}{|l|}{ BCL6 } \\
\hline Positive & 0 & 1 & 0 & 3 & 1 & 0 & 3 & 8 & 6.8 \\
\hline \multicolumn{10}{|c|}{ pSTAT5 (Y694) } \\
\hline Negative & 2 & 2 & 2 & 0 & 0 & 0 & 3 & 9 & \\
\hline Positive & 31 & 28 & 3 & 4 & 2 & 1 & 39 & 108 & 92.3 \\
\hline Negative & 1 & 0 & 1 & 0 & 0 & 0 & 5 & 7 & \\
\hline Positive & 32 & 30 & 4 & 4 & 2 & 1 & 37 & 110 & 94.0 \\
\hline Total & 33 & 30 & 5 & 4 & 2 & 1 & 42 & 117 & \\
\hline
\end{tabular}

HeH, high hyperdiploid; KMT2A, KMT2A-rearranged; Ph, Philadelphia chromosome (BCR-ABL1).

We evaluated co-expression of the mRNA for the pre-BCR genes (IGLL1, IGLL3, VPREB1, VPREB3, IGHM, SYK and $Z A P 70)$ and $B C L 6$ by discretising the gene expression to high versus low/non-expressed but did not find significant association between discrete expression groups or correlation between BCL6 and the pre-BCR 'metagene' in the two different datasets ${ }^{19-21}$ (Supplementary Fig. 1 and 2, Appendix A). We noted that BCL6 mRNA did not show a clearly separable positive population in its signal distribution in any of the samples analysed. However, the pre-BCR 'metagene' expression was higher in the TCF3-PBX1 and $M E F 2 D$ subtypes (Mann-Whitney U-test $p<0.001$ ), and $B C L 6$ expression was higher in the cases with high pre-BCR 'metagene' expression (highest $10 \%$ quantile, Mann-Whitney U-test $p<0.001$ ).

Taken together, our results indicate that both on protein and mRNA level, BCL6 alone may not distinguish the patient group with concomitant pre-BCR signalling.

\section{Expression of $B C L 6$ across haematological malignancies and B-ALL subtypes}

BCL6 plays an essential role in B-cell maturation and has been associated with various haematological malignancies. ${ }^{8}$ To obtain a comprehensive picture, we evaluated the expression of BCL6 mRNA across 36 haematological disease entities, cancer cell lines and healthy cells utilising the Hemap dataset. ${ }^{19,20}$ As shown in Fig. 2A, expression of BCL6 mRNA was evident in B-cell malignancies, including acute leukaemia and lymphoma, and T-cell acute lymphoblastic leukaemia, while other entities, such as adult T-cell leukaemia/lymphoma, myeloid leukaemia and multiple myeloma, showed lower levels of expression. Among BALL, high hyperdiploid and KMT2A subtypes showed the weakest expression, whereas the $B C R-A B L 1, T C F 3-P B X 1$ and 'other' subtypes had the highest levels of expression (Kruskal-Wallis $p<0.001$ ) (Fig. 2B).

We retrieved two RNA-sequencing datasets that also included novel subtypes of B-ALL. ${ }^{21,22}$ In addition to the well known subtypes (TCF3-PBX1, ETV6-RUNX1), several novel groups of ALL showed strong expression of the BCL6 mRNA including the Ph-like, MEF2D, NUTM1-rearranged (NUTM1) and PAX5-altered (PAX5-alt) subgroups (Fig. 2C). Taken together, our analysis of mRNA expression captured the expected specificity of BCL6 expression at disease and subtype levels, with the exception of MLL that showed weak expression in the microarray profiles.

\section{High BCL6 mRNA is associated with favourable EFS in B-ALL}

Previously, high expression of BCL6 mRNA has been associated with inferior prognosis in high risk patient cohorts that included both children and adults. ${ }^{9,13,15,16}$ To ascertain the prognostic value of expression of the BCL6 mRNA in a paediatric setting, we evaluated EFS in the Nordic patient cohort by using the median expression of BCL6 mRNA as a cut-off. In this setup, cases with higher-than-median expression of $B C L 6$ had better EFS (log-rank test $p=0.03$ ) (Fig. 3), but it did not translate into better OS (data not shown). In the univariate Cox regression model, higher-than-median BCL6 mRNA was associated with a lower HR $[0.46,95 \%$ confidence interval (CI) $0.22-0.94, p=0.03]$. In the multivariate model, higherthan-median BCL6 mRNA showed a similar trend towards decreased HR $(0.47,95 \%$ CI $0.21-1.04, p=0.06)$ (Table 3).

We further evaluated the impact of the BCL6 protein expression to EFS and OS by using the dataset with immunohistochemistry data, but no statistically significant difference was noted between the BCL6-positive and -negative cases (Supplementary Fig. 3, Appendix A). Similarly, in the Cox univariate model, BCL6 expression did not show a statistically significant effect on OS or EFS. The association of BCL6 expression with diagnostic clinical findings (WBC and blast count, CNS disease), therapy response (MRD at EOI) and major events (relapse, death, secondary malignancy) did not reveal statistically significant findings. Similarly, positivity for either ZAP70 or pSTAT5 did not associate significantly with the patient survival (data not shown).

\section{DISCUSSION}

BCL6 is a transcriptional repressor that is necessary for germinal centre formation and antibody affinity maturation. It has a well established role in several mature B-cell malignancies, and recent studies have implicated a role in BALL as well. ${ }^{9,23}$ We report here that approximately $7 \%$ of childhood B-ALL cases were positive for the BCL6 protein. High $B C L 6$ mRNA levels were most common among the TCF3-PBX1, Ph-like, NUTM1, MEF2D and PAX5-alt subgroups, and higher-than-median expression of BCL6 was associated with favourable EFS.

Gene expression has been widely studied as a tool to improve classification of disease entities. ${ }^{21,24}$ However, mRNA expression does not correlate fully with protein expression, ${ }^{25-28}$ and therefore we collected a retrospective 
A

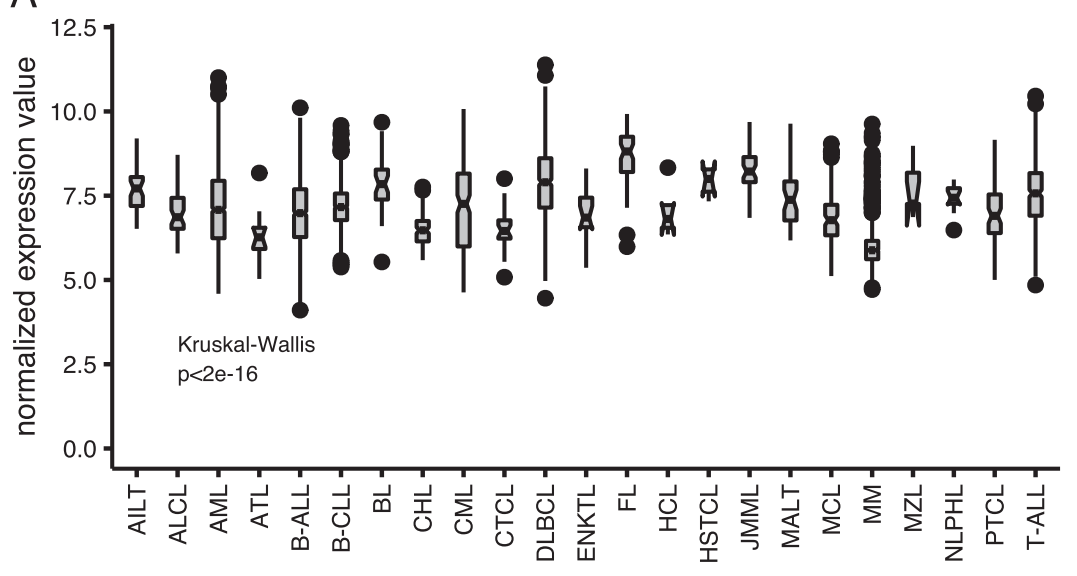

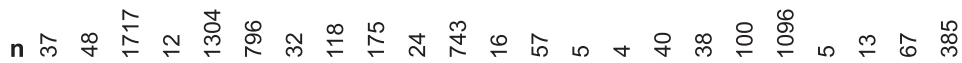

B

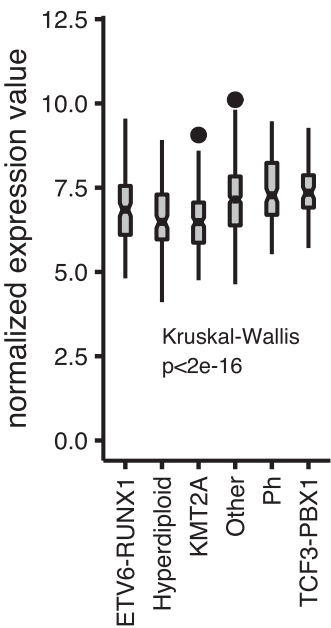

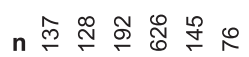

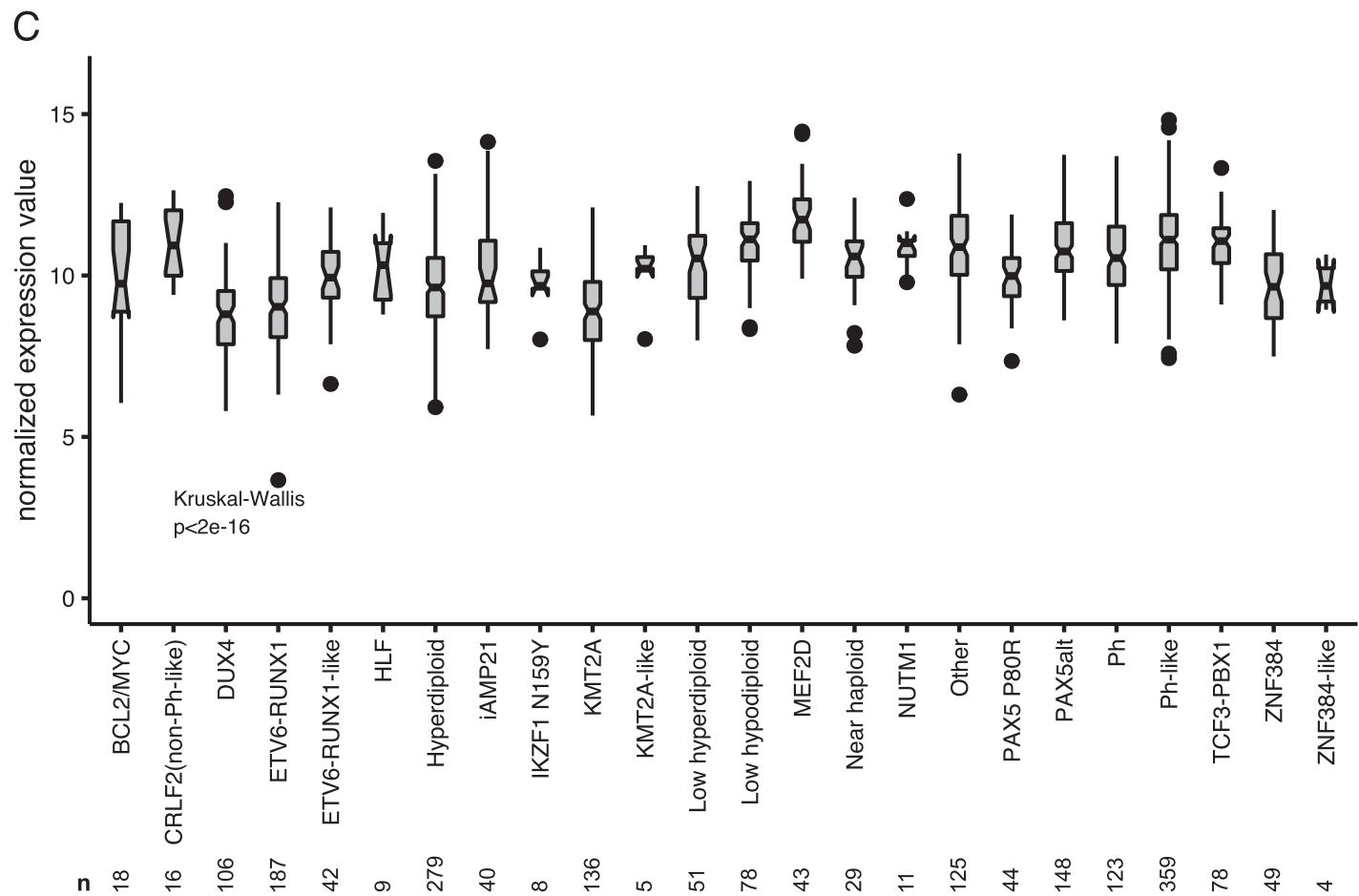

Fig. 2 Expression of $B C L 6$ mRNA in haematological malignancies and B-cell lineage acute lymphoblastic leukaemia (B-ALL). (A) Boxplot showing expression of the $B C L 6$ mRNA in different haematological malignancies in the Hemap dataset $(n=6832){ }^{19,20}$ (B) Boxplot showing expression of $B C L 6$ mRNA in the main subtypes of BALL in the Hemap dataset $(n=1304)$. (C) Boxplot showing expression of the BCL6 mRNA in different subtypes of B-ALL in the PanALL study dataset $(n=1988)$. ${ }^{21}$ AILT, angioimmunoblastic T-cell lymphoma; ALCL, anaplastic large cell lymphoma; AML, acute myeloid leukaemia; ATL, adult T-cell leukaemia; B-ALL, B-cell lineage acute lymphoblastic leukaemia; B-CLL, B-cell chronic lymphocytic leukaemia; BCL2/MYC, BCL2/MYC rearranged; BL, Burkitt lymphoma; CHL, classic Hodgkin lymphoma; CML, chronic myeloid leukaemia; CRLF2, CRLF2 (non-Ph-like); CTCL, cutaneous T-cell lymphoma; DLBCL, diffuse large B-cell lymphoma; DUX4, DUX4-rearranged; ENKTL, extranodal NK/T-cell lymphoma; FL, follicular lymphoma; HCL, hairy cell leukaemia; HLF, TCF3/TCF4-HLF; HSTCL, hepatosplenic T-cell lymphoma; iAMP21, intrachromosomal amplification of chromosome 21; IKZF1 N159Y, IKZF1 missense alteration encoding p.Asn159Tyr; JMML, juvenile myelomonocytic leukaemia; KMT2A, KMT2A rearranged; MALT, extranodal marginal zone lymphoma of mucosa-associated lymphoid tissue; MCL, mantle cell lymphoma; MEF2D, MEF2D rearranged; MM, multiple myeloma; MZL, marginal zone lymphoma; n, number of cases; NLPHL, nodular lymphocyte predominant Hodgkin lymphoma; NUTM1, NUTM1 rearranged; PAX5alt, PAX5 alterations; PAX5 P80R, PAX5 p.Pro80Arg (P80R) alteration; Ph, Philadelphia chromosome (BCRABL1); PTCL, peripheral T-cell lymphoma, not otherwise specified; T-ALL, T-cell lineage acute lymphoblastic leukaemia; ZNF384, ZNF384 rearranged. 


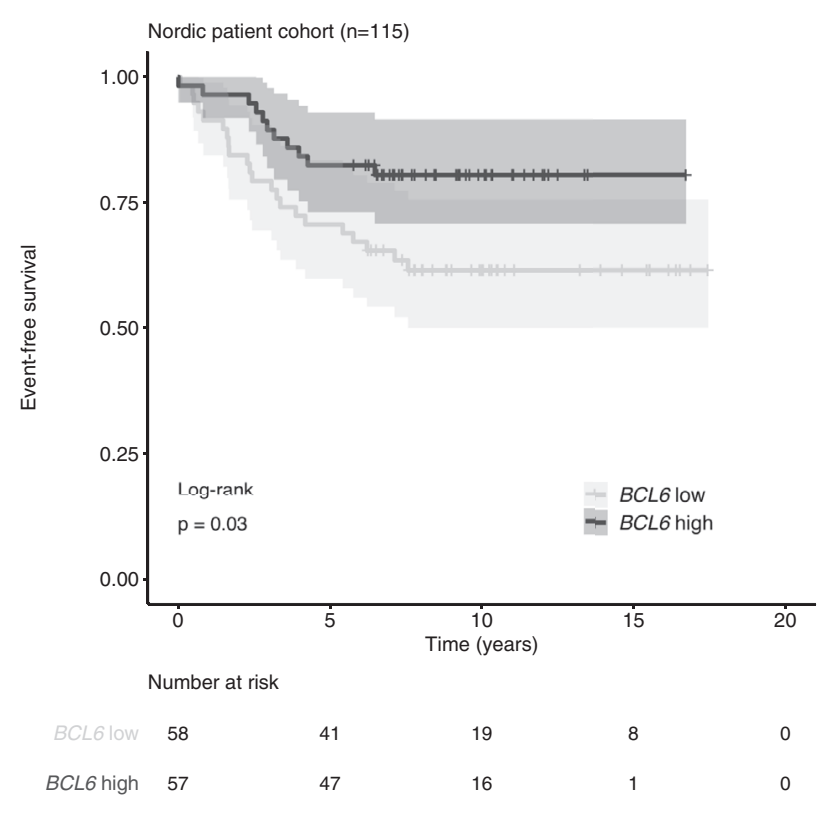

Fig. 3 Association of BCL6 expression with the survival of patients. Kaplan-Meier survival curve and log-rank test $p$ value for EFS analyses comparing EFS in cases with higher- or lower-than-median BCL6 mRNA in the Nordic patient cohort with mRNA sequencing data $(n=115) .{ }^{22}$

patient cohort that had archived trephine biopsy samples. Our cohort was population based and represented the most common subtypes of B-ALL according to the WHO 2017 classification. ${ }^{17}$ We note that knowledge of Ph-like, IGH/IL3rearranged B-ALL and iAMP21 B-ALL was lacking in our biopsy cohort, as novel subtypes have been identified only during the last few years. These cases were likely among the 'other' group of B-ALL in our cohort.

BCL6 protein expression was found in $7 \%$ of paediatric BALL cases, which is lower than the $12-17 \%$ previously reported. ${ }^{9,10,13}$ Previous sample cohorts with BCL6 protein expression were composed of both paediatric and adult patients. ${ }^{9,10,13}$ In addition, the published cohorts had fewer patients (52-72 patients) and used BM aspirate clot specimens, in contrast to the formalin fixed BM trephine biopsies in our study. In order to ensure the quality of the analysis, two pathologists independently evaluated the immunohistochemically stained samples, and the discrepant cases were solved by a third pathologist. The decalcification process or the used antibody, which was different from the one used in previous studies, might have affected the efficiency of the immunohistochemistry, thereby decreasing the number of positive cases in our cohort. ${ }^{9,10,13,29}$ However, three Burkitt leukaemia/lymphoma cases, which were used as the positive controls, were all BCL6 positive as expected, suggesting that neither the antibody nor the sample processing were behind the low prevalence of BCL6 positivity.

High BCL6 has been suggested to represent the more differentiated precursor B-cell leukaemia than the progenitortype B-cell leukaemia. ${ }^{9}$ However, co-immunostaining of BCL6 with either the ZAP70 (pre-B-ALL) or phosphorylated STAT5 (pro-B-ALL) antibody did not show a clear dichotomy, despite the fact that the proportions of positivity to ZAP70, phosphorylated STAT5 and BCL6 were roughly in line with the previous literature. ${ }^{9}$ It is possible that the sensitivity of the antibody-based detection of active signalling pathways by IHC is not as good as the multiparametric flow cytometry-based assay, ${ }^{30}$ and could explain the low co-expression. On the other hand, mRNA expression of the pre-BCR+ 'metagene', 9,14 which was associated with the TCF3-PBXI and $M E F 2 D$ subtypes in two different datasets, did not correlate with the $B C L 6$ expression. ${ }^{14}$ However, BCL6 mRNA expression was statistically significantly higher in the $10 \%$ of cases with highest pre-BCR 'metagene' expression. In addition, BCL6 protein expression did associate with the CD34-negative immunophenotype in line with the previous literature. 9,10 Combined, these results suggest that an association exists between pre-BCR-signalling and BCL6, but there is more heterogeneity in signalling pathways in BCL6-positive B-ALL than earlier reported. Thus, it is possible that other routes for activation of BCL6 exist, or the cell population is heterogeneous with respect to signalling pathway activity.

Table 3 Cox proportional hazards models for survival in the Nordic patient cohort $(n=115)^{22}$

\begin{tabular}{|c|c|c|c|c|c|c|c|}
\hline & \multirow[t]{2}{*}{$n$} & \multicolumn{3}{|c|}{ Multivariate Cox regression } & \multicolumn{3}{|c|}{ Univariate Cox regression } \\
\hline & & HR & $95 \% \mathrm{CI}$ & $p$ & HR & $95 \% \mathrm{CI}$ & $p$ \\
\hline \multicolumn{8}{|l|}{ Age } \\
\hline 1 year & 7 & 1.0 & & & 1.00 & & \\
\hline $1-10$ years & 80 & 0.38 & $0.08-1.94$ & 0.25 & 0.30 & $0.10-0.87$ & $\mathbf{0 . 0 3}$ \\
\hline$>10$ years & 28 & 0.31 & $0.06-1.74$ & 0.19 & 0.36 & $0.11-1.19$ & 0.09 \\
\hline \multicolumn{8}{|l|}{ WBC } \\
\hline$\leq 50 \times 10^{9} / \mathrm{L}$ & 101 & 1.00 & & & 1.00 & & \\
\hline$>50 \times 10^{9} / \mathrm{L}$ & 14 & 0.63 & $0.16-2.54$ & 0.52 & 1.88 & $0.78-4.56$ & 0.16 \\
\hline \multicolumn{8}{|l|}{ WHO subgroup } \\
\hline Other & 55 & 1.00 & & & 1.00 & & \\
\hline $\mathrm{HeH}$ & 42 & 0.72 & $0.31-1.67$ & 0.45 & 0.63 & $0.29-1.36$ & 0.24 \\
\hline ETV6-RUNX1 & 18 & 0.89 & $0.28-2.82$ & 0.84 & 0.58 & $0.20-1.71$ & 0.32 \\
\hline \multicolumn{8}{|l|}{ Treatment } \\
\hline Standard risk & 32 & 1.00 & & & 1.00 & & \\
\hline Other & 83 & 1.85 & $0.70-4.84$ & 0.21 & 1.94 & $0.80-4.70$ & 0.14 \\
\hline High $(>\mathrm{md})$ & 57 & 0.47 & $0.21-1.04$ & 0.06 & 0.46 & $0.22-0.94$ & $\mathbf{0 . 0 3}$ \\
\hline Total $n$ & 115 & & & & & & \\
\hline
\end{tabular}

CI, confidence interval; HeH, high hyperdiploid; HR, hazard ratio; md, median; WBC, white blood cell count.

Statistically significant $p$ values $<0.05$ in bold. 
To further evaluate the expression of $B C L 6$, we retrieved three independent microarray or RNA-sequencing datasets. High levels of BCL6 mRNA were associated with the TCF3$P B X 1$, Ph-like, NUTM1, and PAX5-alt subgroups. Also, expression of BCL6 mRNA was prominent in the newly described $M E F 2 D$ subtype, and fittingly, Tsuzuki et al. recently identified SREBF1 as a possible drug target in this subtype. ${ }^{14}$ In contrast, we could not show an association with the $K M T 2 A$ (formerly MLL) subtype, a finding recently reported by Hurtz et al. ${ }^{13}$

MuHC has been proposed as an additional biomarker for the pre-BCR+ ALL subgroup. ${ }^{9}$ We tested muHC immunohistochemistry in the trephine biopsy samples, but strong background staining hampered the evaluation, suggesting that the accurate detection of muHC expression might be more suitable from clot samples and flow cytometry than the trephine biopsy specimens.

The association of BCL6 protein expression on survival has not been previously investigated in a paediatric B-ALL cohort. In our local cohort, we did not notice a statistically significant difference in survival based on BCL6 positivity, possibly due to rarity of its occurrence. In contrast, higher-than-median expression of BCL6 mRNA was associated with favourable EFS in a Nordic patient cohort and showed a similar trend in the multivariate model. Restricting the analysis to the strongest expressors only (top 10\%) did not change the result. Unfortunately, we lacked the mRNA data for the biopsy cohort in order to determine the mRNA threshold when the protein expression turns positive in biopsy samples (although the association is likely not linear). Our results do not indicate that BCL6 protein or mRNA levels alone would associate with an inferior prognosis as suggested by earlier literature., $93,15,16$ These discrepancies may relate to the differences in the composition of the patient cohorts and the thresholding used. Instead, based on the results obtained, a combination of protein markers, or the pre-BCR 'metagene' may better stratify the patient group referred to as pre-BCR+ ALL.

In summary, BCL6 is associated with distinct diagnostic and novel subgroups of B-ALL. BCL6 expression is widely used as a diagnostic and classification tool in B- and T-cell lymphomas and Hodgkin lymphomas. ${ }^{17}$ In B-ALL, its utility as a biomarker for pre-BCR+ warrants further studies with co-staining of additional markers and parallel transcriptome profiling in larger patient cohorts to distinguish patients that may benefit from its therapeutic targeting.

Acknowledgements: The authors wish to thank Eini Eskola for assistance in laboratory work.

Conflicts of interest and sources of funding: The work was supported by Competitive State Research Financing of the Expert Responsibility at Tampere University Hospital (9X027) and grants from the Academy of Finland (no. 277816 and 310106, OL), Sigrid Jusélius Foundation (MH and OL), the Cancer Society of Finland (MH, OL), the Jane and Aatos Erkko Foundation (OL, MH) and the Väre Foundation for paediatric Cancer Research (AM). The authors declare no competing interests.

\section{APPENDIX A. SUPPLEMENTARY DATA}

Supplementary data to this article can be found online at https://doi.org/10.1016/j.pathol.2021.02.013.
Address for correspondence: Artturi Mäkinen, MD, Tampere University, Arvo Ylpön katu 34, PO Box 100, FI-33014, Tampere, Finland. E-mail: artturi.makinen@tuni.fi

\section{References}

1. Inaba H, Greaves M, Mullighan CG. Acute lymphoblastic leukaemia. Lancet 2013; 381: 1943-55.

2. Hunger SP, Mullighan CG. Acute lymphoblastic leukemia in children. New Eng J Med 2015; 373: 1541-52.

3. Lee D, Grigoriadis G, Westerman D. The role of multiparametric flow cytometry in the detection of minimal residual disease in acute leukaemia. Pathology 2015; 47: 609-21.

4. Ye BH, Cattoretti G, Shen Q, et al. The BCL-6 proto-oncogene controls germinal-centre formation and Th2- type inflammation. Nat Genet 1997 16: $161-70$.

5. Dent AL, Shaffer AL, Yu X, et al. Control of inflammation, cytokine expression, and germinal center formation by BCL-6. Science 1997 276: $589-92$

6. Huang C, Geng H, Boss I, et al. Cooperative transcriptional repression by BCL6 and BACH2 in germinal center B-cell differentiation. Blood 2014; 123: 1012-20.

7. Hatzi K, Melnick A. Breaking bad in the germinal center: how deregulation of BCL6 contributes to lymphomagenesis. Trends Mol Med 2014; 20: $343-52$.

8. Cardenas MG, Oswald E, Yu W, et al. The expanding role of the BCL6 oncoprotein as a cancer therapeutic target. Clin Cancer Res 2017; 23: $885-93$.

9. Geng H, Hurtz C, Lenz KB, et al. Self-enforcing feedback activation between BCL6 and pre-B cell receptor signaling defines a distinct subtype of acute lymphoblastic leukemia. Cancer Cell 2015; 27 409-25.

10. Deucher AM, Qi Z, Yu J, et al. BCL6 expression correlates with the $\mathrm{t}(1 ; 19)$ translocation in B-lymphoblastic leukemia. Am J Clin Pathol 2015; 143: 547-57.

11. Köhrer S, Havranek O, Seyfried F, et al. Pre-BCR signaling in precursor B-cell acute lymphoblastic leukemia regulates PI3K/AKT, FOXO1 and MYC, and can be targeted by SYK inhibition. Leukemia 2016; 30: $1246-54$.

12. Kim E, Hurtz C, Koehrer S, et al. Ibrutinib inhibits pre-BCR+ B-cell acute lymphoblastic leukemia progression by targeting BTK and BLK. Blood 2017; 129: 1155-65.

13. Hurtz C, Chan LN, Geng H, et al. Rationale for targeting BCL6 in MLLrearranged acute lymphoblastic leukemia. Genes Dev 2019; 33: $1265-79$.

14. Tsuzuki S, Yasuda T, Kojima S, et al. Targeting MEF2D-fusion oncogenic transcriptional circuitries in B-cell precursor acute lymphoblastic leukemia. Blood Cancer Discov 2020; 1: 82-95.

15. Swaminathan $\mathrm{S}$, Huang $\mathrm{C}$, Geng $\mathrm{H}$, et al. BACH2 mediates negative selection and p53-dependent tumor suppression at the pre-B cell receptor checkpoint. Nat Med 2013; 19: 1014-22.

16. Ge Z, Zhou X, Gu Y, et al. Ikaros regulation of the BCL6/BACH2 axis and its clinical relevance in acute lymphoblastic leukemia. Oncotarget 2017; 8: 8022-34.

17. Swerdlow SH, Campo E, Harris NL, et al. WHO Classification of Tumours of Haematopoietic and Lymphoid Tissues. Revised 4th ed. Lyon IARC, 2017.

18. Grönroos T, Mäkinen A, Laukkanen S, et al. Clinicopathological features and prognostic value of SOX11 in childhood acute lymphoblastic leukemia. Sci Rep 2020; 10: 1-12.

19. Pölönen P, Mehtonen J, Lin J, et al. Hemap: an interactive online resource for characterizing molecular phenotypes across hematologic malignancies. Cancer Res 2019; 79: 2466-79.

20. Mehtonen J, Pölönen P, Häyrynen S, et al. Data-driven characterization of molecular phenotypes across heterogeneous sample collections. Nucleic Acids Res 2019; 47: 76.

21. Gu Z, Churchman M, Roberts K, et al. PAX5-driven subtypes of Bprogenitor acute lymphoblastic leukemia. Nat Genet 2019; 51: 296-307.

22. Marincevic-Zuniga Y, Dahlberg J, Nilsson S, et al. Transcriptome sequencing in pediatric acute lymphoblastic leukemia identifies fusion genes associated with distinct DNA methylation profiles. J Hematol Oncol 2017; 10: 148.

23. Chan LN, Murakami MA, Robinson ME, et al. Signalling input from divergent pathways subverts B cell transformation. Nature 2020; 583: 845-51.

24. Brown LM, Lonsdale A, Zhu A, et al. The application of RNA sequencing for the diagnosis and genomic classification of pediatric acute lymphoblastic leukemia. Blood Adv 2020; 4: 930-42. 
25. Csárdi G, Franks A, Choi DS, et al. Accounting for experimental noise reveals that mRNA levels, amplified by post-transcriptional processes, largely determine steady-state protein levels in yeast. PLoS Genet 2015; 11: e1005206.

26. Liu Y, Beyer A, Aebersold R. On the dependency of cellular protein levels on mRNA abundance. Cell 2016; 165: 535-50.

27. Latonen L, Afyounian E, Jylhä A, et al. Integrative proteomics in prostate cancer uncovers robustness against genomic and transcriptomic aberrations during disease progression. Nat Commun 2018; 9: $1-13$.
28. Sjöstedt E, Zhong W, Fagerberg L, et al. An atlas of the proteincoding genes in the human, pig, and mouse brain. Science 2020; 367: eaay5947.

29. Miquelestorena-Standley E, Jourdan ML, Collin C, et al. Effect of decalcification protocols on immunohistochemistry and molecular analyses of bone samples. Mod Pathol 2020; 33: 1505-17.

30. Covey TM, Cesano A. Modulated multiparametric phosphoflow cytometry in hematological malignancies: technology and clinical applications. Best Pract Res Clin Haematol 2010; 23: 319-31. 